\title{
Research Paper: New Biomarkers Based on Smoking- Related Phenotypes for Smoking Cessation Outcomes of Nicotine Replacement Therapy: A Prospective Study
}

\author{
Arash Nikkholgh ${ }^{1}$ (D), Soltan Ahmad Ebrahimi ${ }^{2}$ (D), Enayatollah Bakhshi ${ }^{3}$ (D), Mohammad-Reza Zarrindast ${ }^{4,5}$ (D), Yazdan Asgari ${ }^{6}$, Anahita \\ Torkaman-Boutorabi ${ }^{17^{* *}}$ \\ 1. Department of Neuroscience and Addiction Studies, School of Advanced Technologies in Medicine, Tehran University of Medical Sciences, Tehran, Iran. \\ 2. Department of Pharmacology, School of Medicine, Iran University of Medical Sciences, Tehran Iran. \\ 3. Department of Biostatistics and Epidemiology, University of Social Welfare and Rehabilitation Sciences, Tehran, Iran. \\ 4. Iranian National Center for Addiction Studies, Tehran University of Medical Sciences, Tehran, Iran. \\ 5. Department of Pharmacology, School of Medicine, Tehran University of Medical Sciences, Tehran, Iran. \\ 6. Department of Medical Biotechnology, School of Advanced Technologies in Medicine, Tehran University of Medical Sciences, Tehran, Iran \\ 7. Research Center for Cognitive and Behavioral Studies, Tehran University of Medical Sciences, Tehran, Iran
}

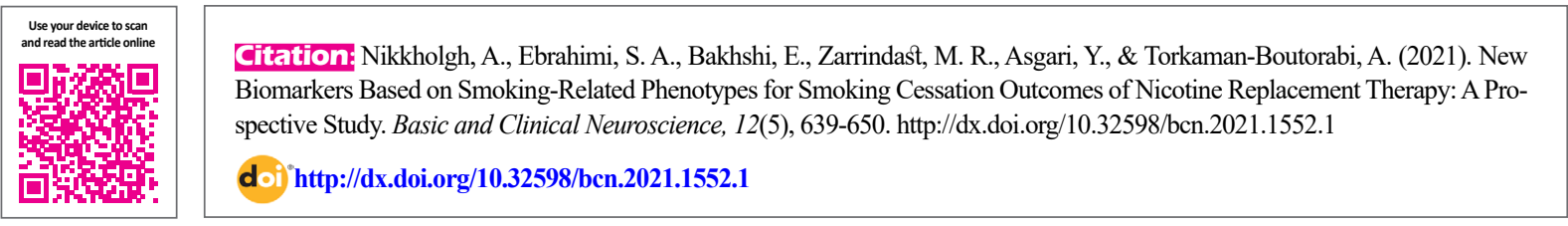

\section{(1) (\$)}

Article info:

Received: 23 Sep 2020

First Revision: 02 Jun 2021

Accepted: 28 Aug 2021

Available Online: 01 Sep 2021

Keywords:

Cotinine, Carbon monoxide,

Nicotine replacement therapy,

Smoking cessation

\section{AB S T RACT}

Introduction: Identifying a potent biomarker for smoking cessation can play a key role in predicting prognosis and improving treatment outcomes. This study aimed to evaluate the contribution of new biomarkers based on the levels of Cotinine (Cot) and carbon monoxide (CO) to the short- and long-term quit rates of nicotine replacement therapies (Nicotine Patch $[\mathrm{NP}]$ and Nicotine Lozenge [NL]).

Methods: In this prospective interventional study, 124 smokers under treatment with the 5A's method were selected from an outpatient smoking cessation center in district 18 of Tehran City, Iran. The study was conducted from April 2016 to December 2018. They were divided into NP $(\mathrm{n}=56)$ and $\mathrm{NL}(\mathrm{n}=61)$ intervention groups. The levels of Cot and $\mathrm{CO}$ were measured using ELISA and breath analysis at the beginning of the study. Three markers were calculated: Cot/ $\mathrm{CO}$, Cot to cigarette per day ratio $(\mathrm{Cot} / \mathrm{CPD})$, and $\mathrm{CO} / \mathrm{CPD}$. Binary logistic regression models and generalized estimating equations models were analyzed by SPSS software, version 21 to determine the chances of quitting smoking.

Results: Of the NP participants, 30.4\% and 19.6\% were abstinent after 2 and 6 months, respectively, while NL was found less effective with $19.7 \%$ for 2-month follow-up and $13.1 \%$ for 6-month follow-up. The 6-month success of quitting attempts was significantly different for the NP participants at the second half of $\mathrm{Cot} / \mathrm{CO}(\mathrm{P}=0.029)$. Of the NL participants, $\mathrm{CO} / \mathrm{CPD}$ would be a superior predictor for smoking cessation success $(\mathrm{P}>0.05)$.

Conclusion: The findings of this study suggested two markers of $\mathrm{Cot} / \mathrm{CO}$ and $\mathrm{CO} / \mathrm{CPD}$ in this order for the optimum treatment outcomes of NP and NL.

\section{* Corresponding Author:}




\section{Highlights}

- Smoking is one of the significant reasons deaths across the globe.

- There were some smoking cessation approaches, including behavioral or pharmacological interventions.

- The rate of treatment failure is considerable.

\section{Plain Language Summary}

Tobacco-induced diseases, namely chronic obstructive pulmonary disease, cancer, and cardiovascular disease, largely causes deaths in the world. Identifying a potent biomarker for smoking cessation can play a key role in predicting prognosis and improving treatment outcomes. This study aimed to evaluate the contribution of new biomarkers based on the levels of Cotinine (Cot) and carbon monoxide (CO) to the short- and long-term quit rates of nicotine replacement therapies. The genotypes of the enzymes involved in metabolic phases play an essential role in phenotypes, such as the rate of nicotine metabolism, the serum level of cotinine, and the metabolite of nicotine. studies have explored many biomarkers, such as plasma and urinary levels of nicotine, total nicotine equivalents, and exhaled Carbon Monoxide (CO).

\section{Introduction}

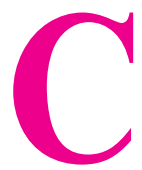

igarette smoking is one of the significant reasons behind many preventable deaths across the globe (Adams \& Morris, 2021; Samet, 2013). Tobacco-induced diseases, namely chronic obstructive pulmonary disease, cancer, and cardiovascular disease, largely account for many premature deaths in the world (Jha, 2020; Saidi et al., 2019). At present, the number of tobacco users is around 1.3 billion smokers, which will rise to 1.6 billion by 2025 (World Health Organization, 2019). The Iran Ministry of Health has reported that the number of cigarettes consumed exceeds 50 billion per year. More importantly, the burden of disease and treatment is associated with skyrocketing health care costs (Alimohammadi et al., 2017; Rezaei, Pulok, \& Ebrahimi, 2020).

There have been some practical smoking cessation approaches, including behavioral or pharmacological interventions. However, the treatment failure rate is considerable, and many smokers cannot succeed even with extra support (Cahill, Lindson-Hawley, Thomas, Fanshawe, \& Tim Lancaster, 2016; Lancaster \& Stead, 2017). Nicotine Replacement Therapies (NRT) are approved by the United States (US) Food and Drug Administration. One NRT is transdermal Nicotine Patch (NP). It has been found widespread applications in the US (Pierce \& Gilpin, 2002; Jonk et al., 2005) and Europe (West, DiMarino, Gitchell, \& McNeill, 2005), and reported with enhanced cessation rates as opposed to placebo (2008 PHS Guideline Update Panel, 2008). Based on clinical trials, its quit rate hardly goes over 30\% (2008 PHS Guideline
Update Panel, 2008). Another almost new NRT is Nicotine Lozenge (NL), which ameliorates cessation rates compared to placebo (Stead et al., 2008; Shiffman et al., 2002).

The marked individual variability in both clinical response and side effects necessitates the development of novel biomarkers for the detection of smoking status and optimization of pharmacotherapy outcomes (Bough et al., 2013). The genotypes of the enzymes involved in metabolic phases play an essential role in phenotypes, such as the rate of nicotine metabolism, the serum level of cotinine, and the metabolite of nicotine (Rao et al., 2000). So, researchers have shown that heritability contributes significantly to tobacco addiction (Kralikova et al., 2013; Wang, Cho, Xiao, Wajsbrot, \& Park, 2013). The involvement of genetically informed biomarkers in the process of pharmacological treatment potentiates cessation rates (Bough et al., 2013; Dempsey et al., 2004), which implies that the same genetic factors which make an individual prone to nicotine addiction are more likely to lessen the response to pharmacotherapy (Brock, Takeda, Brennan, \& Walton, 2011).

As for smoking consumption, studies have explored many biomarkers, such as plasma and urinary levels of nicotine, total nicotine equivalents, and exhaled Carbon Monoxide (CO) (Hartz et al., 2012). Nicotine is one of such tobacco-specific biomarkers (Jacob \& Byrd, 1999) and primarily undergoes metabolism to Cotinine (Cot) in the liver (Benowitz \& Jacob, 1994). The half-life of Cot ranges between 12 and $20 \mathrm{~h}$ in the body (Hammond \& Leaderer, 1987; Jaakkola \& Jaakkola, 1997), which, in 
turn, enables its detection for several days to one week (Benowitz, 1999; SRNT Subcommittee on Biochemical Verification, 2002). Approximately $10 \%-15 \%$ of Cot is eliminated in the urine, with the remaining being converted to trans-3'-hydroxycotinine and other byproducts (Benowitz, Jacob, Fong, \& Gupta, 1994). Given its slower clearance yet longer half-life, the amount of Cot, determined in the saliva, urine, or serum, is vastly utilized as a biomarker of tobacco smoke exposure (Raja, Garg, Yadav, Jha, \& Handa, 2016). On the other hand, CO is a byproduct produced during tobacco combustion (Man $\mathrm{Ki}$ Ho et al., 2009). CO has a short half-life of around 2-3 h (Perkins, Karelitz, \& Jao, 2013) and can signify the recent exposure to smoking. Put differently, the time of the last cigarette is of utmost importance in this biological method. Moreover, previous reports have indicated that the ratio of carbon monoxide to cotinine $(\mathrm{CO} / \mathrm{Cot})$ can be used as a scale showing the severity of smoking. However, others have pointed out that smokers' exposure to $\mathrm{CO}$, Cot, and other tobacco metabolites is either undervalued or overvalued through the self-declaration of the number of Cigarettes Per Day (CPD) (Joseph et al., 2005). In other words, the objective indexes give more trustworthy information concerning smoking patterns than self-reports (Klesges, Debon, \& Ray, 1995).

Determination of pre-treatment biomarkers among smokers, which may be linked to the cessation of smoking, can help smokers and clinicians to select the types of NRT with effective cessation outcomes. The present study was intended to investigate if previous biomarkers derived from smoking intensity, including $\mathrm{Cot} / \mathrm{CO}$, Cot to Cigarette Per Day ratio (Cot/CPD), and CO/CPD (de Leon et al., 2003; Ho et al., 2009) could predict the short- and long-term quit rates of NP and NL in a large sample of treatment-seeking smokers in Iran. Also, this study explored whether results are in agreement across different operationalization of the biomarkers (i.e., continuous measures or based on the second-half above the median of ratios).

\section{Methods}

\section{Study design and participants}

This prospective interventional study includes 470 male individuals treated with 5A's method of smoking cessation counseling. They were selected from Tehran City, Iran. The recruitment of the participants was conducted among members of a social group called NOSMOKINGNEWS in the Telegram application through an intelligent SMS-based notification system in an outpatient smoking cessation center in District 18 of
Tehran from April 2016 to December 2018. We did not enroll women in the present study because of the marked differences in nicotine metabolism related to the effect of estrogen (Benowitz, Lessov-Schlaggar, Swan, \& Jacob, 2006). The inclusion criteria were the age range between 18 and 65 years old, smoking more than 5 cigarettes per day verified by CO (>7 ppm) (coVita, 2020), having residence in Tehran for at least 6 months, and being interested in cessation treatment. The following factors ruled out the participation: the female gender, use of non-tobacco nicotine-containing products such as electronic cigarettes, use of other quit smoking treatments, history of treatments for substance use disorders, history of drug or alcohol abuse, prohibition of medical use of nicotine due to the history of the liver and kidney diseases, cancer and organ transplantation, history of substantial cardiac dysrhythmias, stroke, angina, heart attack or uncontrolled hypertension, history of major depressive disorder, DSM-IV Axis I psychiatric disorders or suicide risk, intake of antipsychotic, stimulant, opiate, antiarrhythmic, anti-coagulant, or antidepressant medicines with an effect on CYP2A6 activity, and failure to deliver informed consent.

\section{Study procedure}

The study participants commonly received the 5A's (Ask, Advise, Assess, Assist, and Arrange) method, including all strategies and advice that a primary care provider should deliver within 3-5 minutes (Martínez et al., 2017; World Health Organization, 2020). As a result of the exclusion criteria, 346 individuals were excluded from the study. The remaining eligible participants $(n=124)$ signed informed consent forms and were referred to a tobacco cessation clinic in Tehran. The present study procedure followed the Code of Ethics of the World Medical Association (the Declaration of Helsinki). The study was approved by the Ethics Committee of the Tehran University of Medical Sciences (code: IR.TUMS. REC.1394.18.96). All participants could benefit from behavioral therapy, were given educational booklets, and accessed the @NOSMOKINGNEWS website for more information. Also, they were offered one session of smoking cessation counseling for $30 \mathrm{~min}$. Based on Figure 1, the participants were randomly assigned into the nicotine patch (NP) and nicotine lozenge (NL) groups and subsequently underwent the assessment of nicotine dependence by Fagerström Test for Nicotine Dependence (FTND) (Heatherton, Kozlowski, Frecker, \& Fagerstrom, 1991) and the physical examination by an expert physician. The group assignment was carried out using a Microsoft Excel-based random number generator. 
For nicotine replacement therapy, all participants received 5 sessions of individual counseling. The first session with a physician expert in smoking cessation lasted an hour and focused on the harms and benefits of quitting as well as techniques that help the person to choose a day to quit within the next two weeks. On the day of withdrawal, the participant received the second counseling session by a trained counselor via telephone, which focused on the side effects of withdrawal and how to deal with them. During the first four weeks of nicotine replacement therapy, each participant had three telephone sessions with the counselor focusing on preventing relapse. The amount of the drug in each group was based on the FTND results. NPs were administered at $14 \mathrm{mg} / \mathrm{d}$ for those with the FTND score $\leq 6$ for the first 6 weeks, followed by $7 \mathrm{mg} / \mathrm{d}$ for the next 6 weeks. For those with the FTND score between 7 and 10, NL was administered $21 \mathrm{mg} / \mathrm{d}$ followed by $14 \mathrm{mg}$ for the next two weeks and then $7 \mathrm{mg}$ for the final 4 weeks. Participants in the NL group received 9 tablets per day for the first 6 weeks, 5 tablets for weeks 7 to 9 , and 3 tablets for 10 to 12 weeks of the treatment. Those with a low FTND score $(\leq 6)$ received 2-mg tablets, and their peers with a high FTND score (7-10) were treated with $4 \mathrm{mg}$ tablets. Smoking cessation was assessed at the end of 3 and 6 months. In this regard, the exhaled $\mathrm{CO}$ level was measured with piCO+smokerlyzer ${ }^{\circledR}$, and those with a $\mathrm{CO}$ reading of $<7$ ppm confirmed abstinent.

\section{Fagerström Test for Nicotine Dependence (FTND)}

The FTND is composed of 6 items to evaluate gradations in tobacco dependence. Further studies have verified that the FTND establishes good internal consistency, positive association with main smoking variables, such as Cot (Heatherton et al., 1991; Payne, Smith, McCracken, McSherry, \& Antony, 1994), and favorable reliability (Sarbandi et al., 2015).

\section{The level of cotinine in the plasma}

Blood samples were obtained from those who claimed to be nicotine-free for five hours, equivalent to the time needed for converting nicotine to Cot with a longer halflife (17 hours) (Sharma, Sane, Anand, Marimutthu, \& Benegal, 2019). Around $6 \mathrm{~mL}$ of peripheral blood was collected by antecubital venipuncture in ethylenediaminetetraacetic acid (EDTA)-containing tubes. The participant's plasma was then separated by centrifugation at $3000 \times \mathrm{g}$ for $3 \mathrm{~min}$ and kept at $-80^{\circ} \mathrm{C}$. The Cot ELISA kit (Crystal, China) was used to assess Cot in the plasma. Using a microplate reader (Hyperion Inc., USA), the intensity of the color was measured at $415 \mathrm{~nm}$.

\section{Exhaled breath $\mathrm{CO}$ analyzer}

The piCO+smokerlyzer ${ }^{\circledR}$ (Bedfont Scientific, England, UK) was used to measure the exhaled $\mathrm{CO}$ levels with standardized smoking thresholds recommended by the manufacturer for different age categories. At first, the amount of room CO was measured, and the machine's calibration was checked at ambient conditions. All subjects were trained to inhale and hold their breath, with every test lasting $15 \mathrm{~s}$. Then, they thoroughly blew into the device's mouthpiece lightly until a loud sound was displayed, which meant completing the breath-submitting task. The breath analysis was performed again when the difference between the results exceeded $2 \mathrm{ppm}$. The exhaled $\mathrm{CO}$ levels were presented in ppm, and the $\mathrm{CO}$ reading of $>6$ ppm confirmed smoking.

\section{Statistical analysis}

SPSS v. 22. (SPSS Inc., Chicago, IL, USA) was employed for data analysis. Descriptive statistics were indicated in the form of mean, Standard Deviation (SD), and the second-half above the median of ratios. The Mann-Whitney U test was applied to compare the differences of age, smoking parameters, and Cot biomarkers between the NP and NL groups. In this study, three biomarkers were calculated: $\mathrm{Cot} / \mathrm{CO}, \mathrm{Cot} / \mathrm{CPD}$, and $\mathrm{CO} /$ CPD. Additionally, these biomarkers were categorized by the half above the median of ratios, and the quit rate was reported for each half. Binary logistic regression models and Generalized Estimating Equations (GEE) model were developed to estimate the odds of smoking cessation success as a function of the biomarkers.

\section{Results}

The study participants were enrolled from the residence of Tehran, who were at least 18 years old, smoked more than 5 cigarettes per day, and received the 5A's method. Of all participants, $50 \%$ was initially allocated to the NP group, which further lost six patients (three had low $\mathrm{CO}$ readings and three withdrew). The other $50 \%$ were assigned to the NL group, of whom only one declined to continue the study after the baseline assessment (Figure 1). As indicated in Table 1, the subjects were matched in terms of age and smoking status $(\mathrm{P}>0.05)$ (Table 1$)$.

At a 2-month follow-up, 30.4\% $(n=17)$ of the participants in the NP group was confirmed as abstinent, whereas the smoking cessation success rate was lower in the NL group $(n=12,19.7 \%)$. After six months, both groups showed a decrease in the success rate $(n=11$, $19.6 \%$ for the NP group; $n=8,13.1 \%$ for the NL group). 
Table 1. Comparing age and smoking parameters between the NP and NL groups

\begin{tabular}{cccc}
\hline Variables & \multicolumn{2}{c}{ Mean \pm SD } & P \\
\cline { 2 - 4 } & $\begin{array}{c}\text { NP Group } \\
(\mathbf{n}=56)\end{array}$ & $\begin{array}{c}\text { NL Group } \\
(\mathbf{n}=61)\end{array}$ & 0.688 \\
\hline Age, year & $42.39 \pm 11.20$ & $42.57 \pm 8.74$ & 0.897 \\
\hline FTND & $6.20 \pm 0.25$ & $6.25 \pm 1.98$ & 0.203 \\
\hline CPD & $11.79 \pm 5.42$ & $12.70 \pm 5.11$ & 0.758 \\
\hline Cot, $\mathrm{ng} / \mathrm{mL}$ & $77.54 \pm 37.65$ & $90.07 \pm 70.59$ & 0.707 \\
\hline CO, ppm & $15.13 \pm 3.80$ & $15.34 \pm 3.68$ & \\
\hline
\end{tabular}

NP: Nicotine Patch; NL: Nicotine Lozenge; FTND: Fagerström Test for Nicotine Dependence; CPD: Cigarette Per Day; Cot: Cotinine; Co: Carbon monoxide.

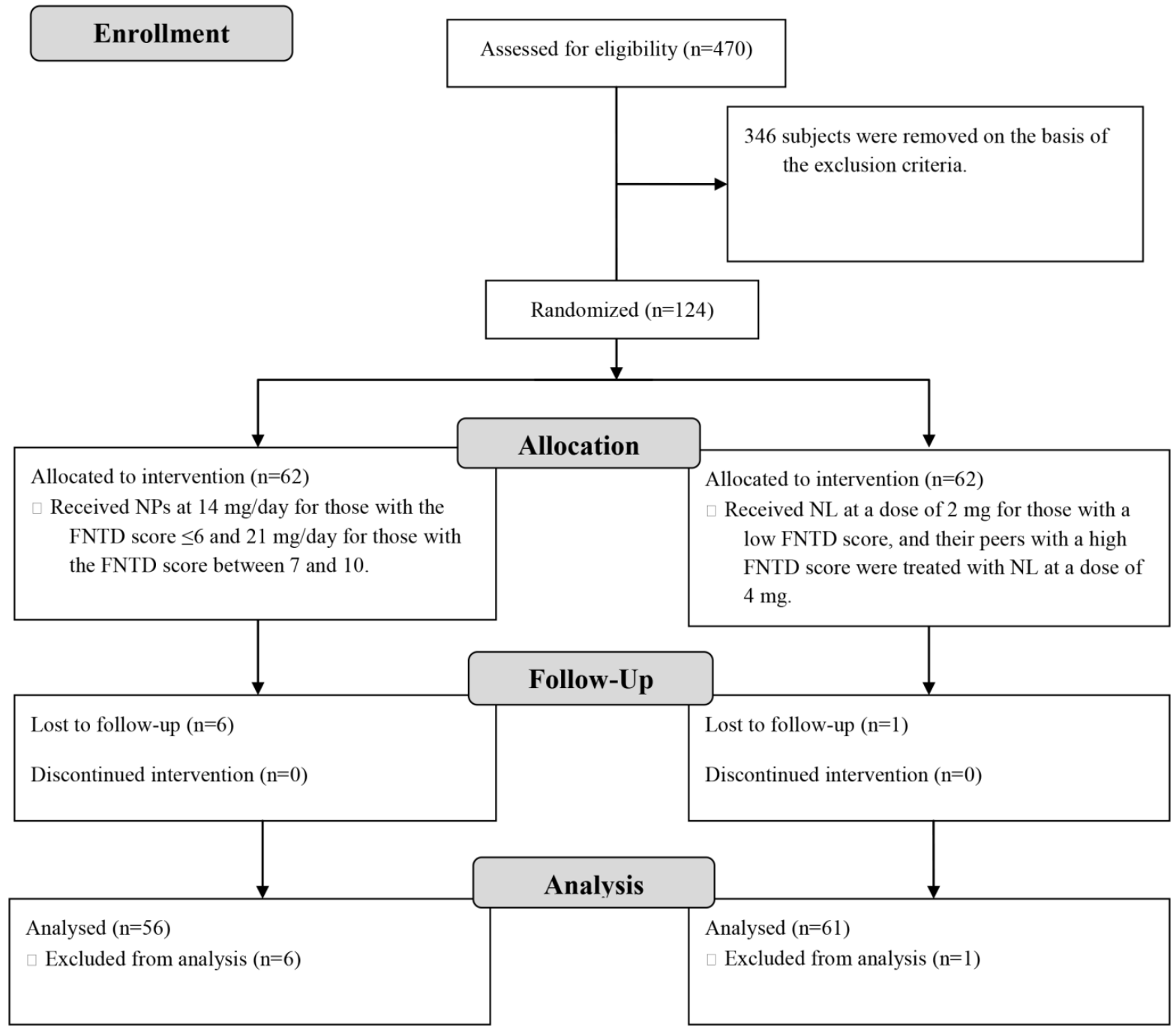

Figure 1. Flow diagram of the subjects through the study

NP: Nicotine Patch; NL: Nicotine Lozenge; FTND: Fagerström Test for Nicotine Dependence. 
Table 2. Cot biomarkers for the study groups

\begin{tabular}{|c|c|c|c|c|c|}
\hline \multirow{2}{*}{ Variables } & \multicolumn{2}{|c|}{ NP Group $(n=56)$} & \multicolumn{2}{|c|}{ NL Group (n=61) } & \multirow{2}{*}{$\mathbf{P}$} \\
\hline & Mean $\pm S D$ & Second Half & Mean $\pm S D$ & Second Half & \\
\hline Cot/CO, ng.mL-1/ppm & $5.16 \pm 0.38$ & 4.52 & $5.37 \pm 0.74$ & 4.72 & 0.881 \\
\hline Cot/CPD, ng.mL-1/d-1 & $7.49 \pm 0.62$ & 7.17 & $7.17 \pm 0.67$ & 7.17 & 0.750 \\
\hline $\mathrm{CO} / \mathrm{CPD}, \mathrm{ppm} / \mathrm{d}-1$ & $1.43 \pm 0.06$ & 1.46 & $1.34 \pm 0.06$ & 1.27 & 0.246 \\
\hline
\end{tabular}

NP: Nicotine Patch; NL: Nicotine Lozenge; CPD: Cigarette Per Day; Cot: Cotinine; Co: Carbon monoxide.

Based on Figure 2, there was no significant difference in the cessation success between the two groups at two or six months $(\mathrm{P}>0.05)$. Table 2 indicates the values of $\mathrm{Cot} / \mathrm{CO}, \mathrm{Cot} / \mathrm{CPD}$, and $\mathrm{CO} / \mathrm{CPD}$. It was found out that these ratios seemed comparable between the NP and NL groups $(\mathrm{P}>0.05)$.

In the NP group, having a Cot/CO ratio above the second half (4.52 ng/mL.ppm) was linked to $39.3 \%$ of the success of attempts to quit after 2 months, and to $28.6 \%$ of the success of attempts to quit after 6 months. As for Cot/CPD, the ratio above the second half $(7.17 \mathrm{ng} . \mathrm{d} / \mathrm{mL})$ meant a minimum success rate of $35.7 \%$ upon 2-month follow-up and 25\% upon 6-month follow-up. When it comes to $\mathrm{CO} / \mathrm{CPD}$, the ratio greater than the second half (1.46 ppm.d) was associated with a minimum success rate of $35.7 \%$ after 2 -month follow-up and $21.4 \%$ after 6-month follow-up (Figure 3).
On the other hand, the NL group exhibited that a Cot/ $\mathrm{CO}$ ratio below the second half (4.72 ng/mL.ppm) led to $20.0 \%$ of the success of attempts to quit after 2 months and $13.3 \%$ of the success of attempts to quit after 6 months. Also, a Cot/CPD ratio less than the second half $(7.17 \mathrm{ng} . \mathrm{d} / \mathrm{mL})$ resulted in a minimum success rate of $23.3 \%$ upon 2-month follow-up and $13.3 \%$ upon 6-month follow-up. In this group, having a $\mathrm{CO} / \mathrm{CPD}$ ratio less than 1.27 ppm.d was linked to a minimum success rate of $24.1 \%$ after 2-month follow-up and $17.2 \%$ after 6-month follow-up (Figure 3).

The result of binomial logistic regression models revealed that only the Cot/CO ratio was significantly (Wald $\left.\chi^{2}=4.761, \mathrm{P}=0.029\right)$ correlated to the 6-month follow-up success rate. When we pooled the quitting at 2-month and 6-month follow-up using the GEE model, there was a significant relationship between the $\mathrm{Cot} / \mathrm{CO}$ ratio and success rate in the NP group $(\mathrm{OR}=1.30,95 \% \mathrm{CI}=1.07$ $1.59, \mathrm{P}<0.05)$. This finding implies that with a 1 unit in-

Table 3. Odds ratios of smoking cessation success from generalized estimating equations models

\begin{tabular}{|c|c|c|c|c|c|c|}
\hline & \multirow{2}{*}{\multicolumn{2}{|c|}{ Variables }} & \multicolumn{2}{|c|}{ The NP Group ( $n=56)$} & \multicolumn{2}{|c|}{ The NL Group ( $n=61$ ) } \\
\hline & & & $\mathrm{OR}^{\mathrm{a}}$ & $95 \% \mathrm{Cl}^{\mathrm{b}}$ & OR & $95 \% \mathrm{Cl}$ \\
\hline \multirow{3}{*}{ Model 1} & & $\mathrm{Cot} / \mathrm{CO}$ & $1.30^{*}$ & $1.07-1.59$ & 0.98 & $0.87-1.10$ \\
\hline & & Following 2 months & 1.00 & \multirow{2}{*}{$0.32-0.86$} & 1.00 & \multirow{2}{*}{$0.39-0.98$} \\
\hline & IIme & Following 6 months & 0.52 & & 0.62 & \\
\hline \multirow{3}{*}{ Model 2} & & Cot/CPD & 1.09 & $0.98-1.22$ & 0.99 & $0.85-1.16$ \\
\hline & & Following 2 months & 1.00 & \multirow{2}{*}{$0.35-0.87$} & 1.00 & \multirow{2}{*}{$0.39-0.98$} \\
\hline & IIme & Following 6 months & 0.54 & & 0.62 & \\
\hline \multirow{3}{*}{ Model 3} & & $\mathrm{CO} / \mathrm{CPD}$ & 1.23 & $0.39-3.90$ & 0.80 & $0.26-2.52$ \\
\hline & & Following 2 months & 1.00 & \multirow{2}{*}{$0.36-0.87$} & 1.00 & \multirow{2}{*}{$0.39-0.98$} \\
\hline & Time & Following 6 months & 0.56 & & 0.62 & \\
\hline
\end{tabular}

NP: Nicotine Patch; NL: Nicotine Lozenge; CPD: Cigarette Per Day; Cot: Cotinine; Co: Carbon monoxide.

adds ratio; ${ }^{\mathrm{b} C o n f i d e n c e ~ i n t e r v a l ; ~}{ }^{*} \mathrm{P}<0.05$. 


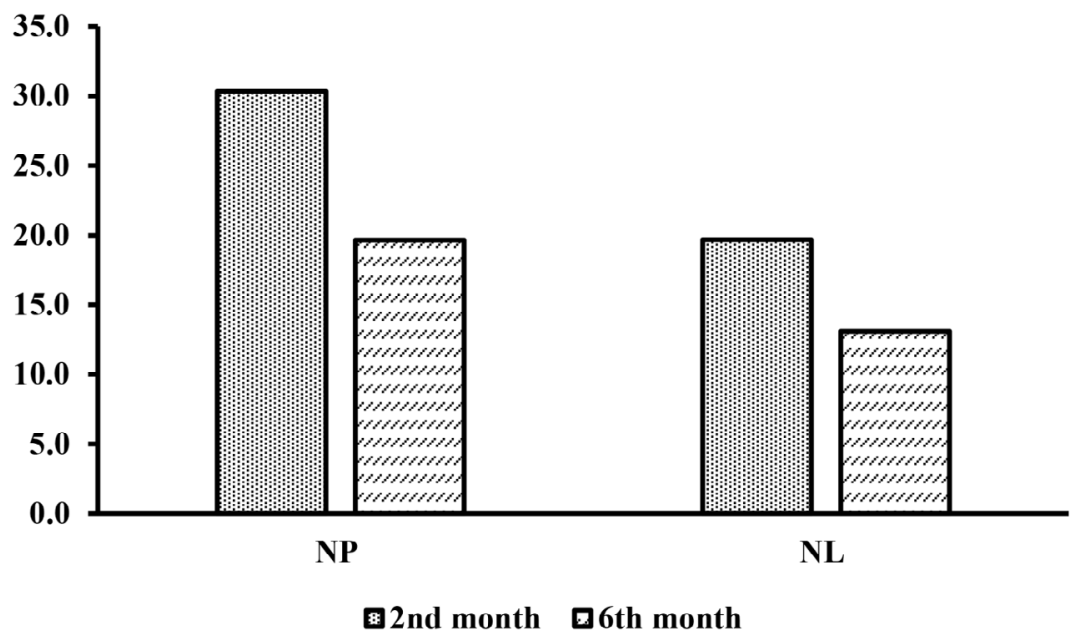

Figure 2. The success of attempts to quit (in percentage) for the study groups

NP: Nicotine patch; NL: Nicotine lozenge.

crease in the Cot/CO, the odds of smoking cessation success were estimated to increase at least $7 \%$ and at most $59 \%$. Notably, the $95 \%$ CI of OR did not include a value one, meaning that a 1 unit increase in the $\operatorname{Cot} / \mathrm{CO}$ would not make any difference in odds. Moreover, all models in Table 3 show that time was inversely associated with the likelihood of smoking cessation success $(\mathrm{OR}=0.52-0.56$ in the NP group; $\mathrm{OR}=0.62$ in the NL group).

\section{Discussion}

Over the past decades, many studies dealing with successful smoking cessation have focused mainly on clinical variables, such as smoking characteristics, demographics, and treatment. Several smoking-related factors contribute to positive cessation outcomes, including lower baseline craving (Waters et al., 2004; Berlin, Singleton, \& Heishman, 2013), the severity of nicotine dependence (Batra, Collins, Torchalla, Schröter, \& Buchkremer, 2008; Paluck et al., 2006), and the number of cigarettes smoked per day (Jay-
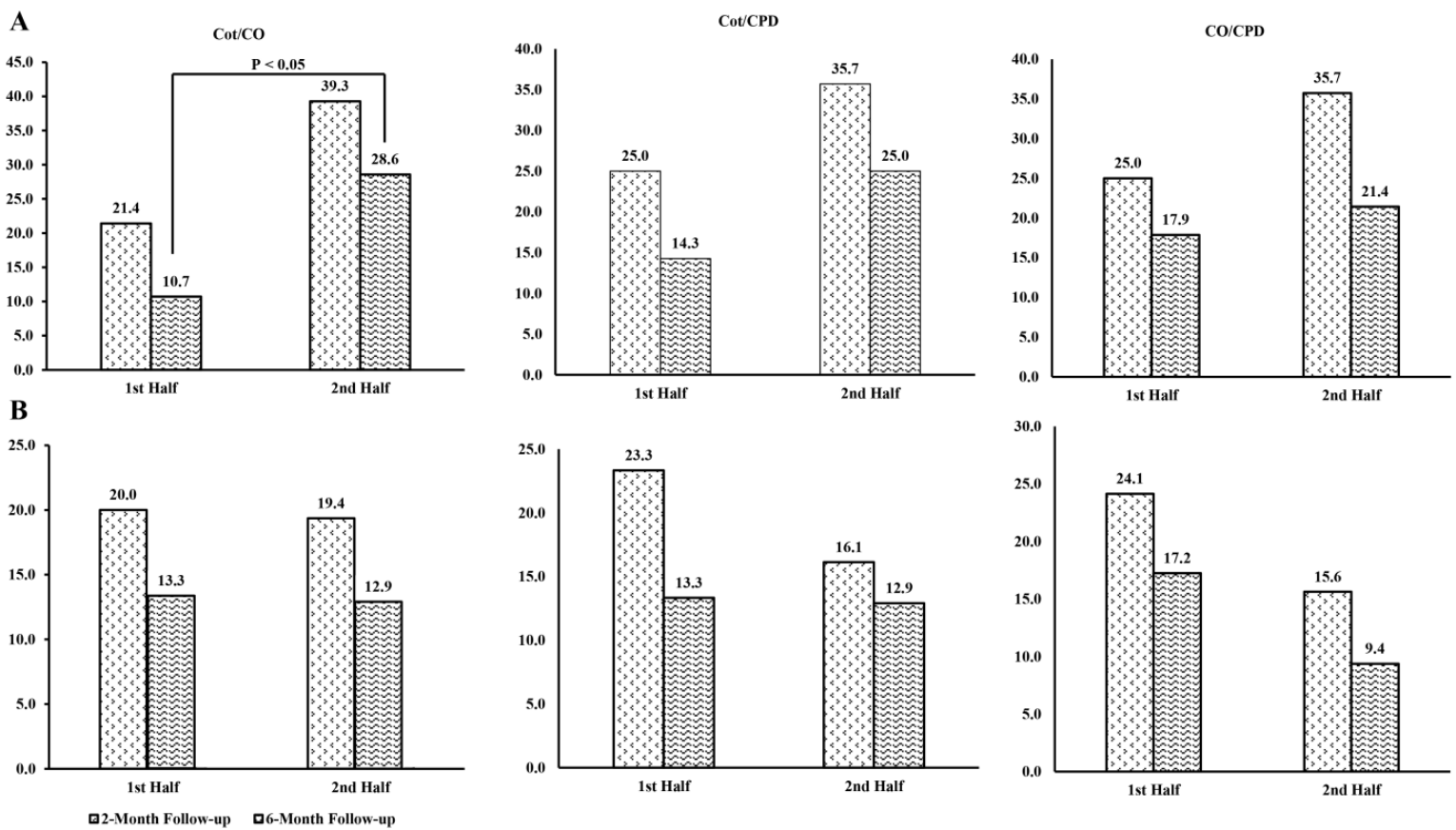

Figure 3. The success of attempts to quit (in percentage) at the second half for the NP (A) and NL (B) Groups

NP: Nicotine patch; NL: Nicotine lozenge. 
akrishnan et al., 2013; Haug, Schaub, \& Schmid, 2014). Recently, the personalization of treatment using validated biomarkers is a new approach to enhance cessation outcomes (Bough et al., 2013). In this case, the Nicotine Metabolite Ratio (NMR) is a good example that exhibits a phenotypic surrogate of nicotine clearance (Dempsey et al., 2004). This biomarker, defined as the ratio of trans3 'hydroxycotinine to Cot, is stable and independent from the time of the last cigarette (Lea, Dickson, \& Benowitz, 2006). However, it differs with sex, race, age, and body mass index (Fogli et al., 2016; Johnstone et al., 2006; Mooney et al., 2008; Rubinstein, Shiffman, Rait, \& Benowitz, 2013). The NMR can also be affected by both environmental inducers and inhibitors, some of which seem transitory (Chenoweth et al., 2014). The present study is intended to propose new biomarkers for different treatment choices and investigate whether smoking cessation time affects the success rates of NP and NL. A crucial step in identifying a new biomarker is to examine if it has effects on smoking cessation in an attempt to improve current smoking cessation treatments.

Our main findings were that the 6-month success rate to quitting was significantly different for the patients under treatment with NP at the second half of the Cot/CO ratio. In other words, the quit rate of NP is more than doubled for the second-highest half of $\operatorname{Cot} / \mathrm{CO}(28.6 \%)$ versus the second-lowest half of $\mathrm{Cot} / \mathrm{Co}(10.7 \%)$. Indeed, this ratio was found helpful in predicting the smoking cessation success and treatment course (at least 6 months) among the NP cases; that is, the Cot/CO ratio greater than 4.52 ng.mL-1/ ppm led to the highest quit rate in the NP group. This result was confirmed by the GEE model. On the contrary, if the patients undergo the NL treatment, the $\mathrm{CO} / \mathrm{CPD}$ ratio will be a superior predictor to the $\mathrm{Cot} / \mathrm{CO}$ ratio. This study showed that the patients who received treatment with NL and had a CO/CPD of less than $1.27 \mathrm{ppm} / \mathrm{d}-1$ experienced the highest quit rate. Therefore, there is room to improve cessation rates by personalizing treatment based on smoking biomarkers (Bough et al., 2013).

These biomarkers can be potentially applied to precision medicine where treatment is tailored based on the susceptibility of patients. In this study, the patients with $\mathrm{Cot} / \mathrm{CO}>4.52$ ng.mL-1/ppm were observed with the highest treatment response to NP, whereas those with $\mathrm{CO} / \mathrm{CPD}<1.27 \mathrm{ppm} / \mathrm{d}-1$ had the highest treatment response to NL. Therefore, there is a risk of treatment failure if related markers are overlooked, and patients face inappropriate treatment. More importantly, the remaining patients (i.e., $\mathrm{Cot} / \mathrm{CO}<4.52$ ng.mL-1/ppm and $\mathrm{CO} /$ $\mathrm{CPD}>1.27 \mathrm{ppm} / \mathrm{d}-1$ ) ran the highest risk of NRT treatment failure.
As for the comparison of the treatments, the smoking cessation success rate was higher in the NP group, and cessation time reduced the success rate in general. For instance, around one-third of the NP group achieved smoking cessation after 2 months, which subsequently reduced to almost one-fifth after 6 months. Similarly, Schnoll et al. reported that quit rates were higher for transdermal nicotine vs nicotine lozenge at the end of treatment and after 6 months (Schnoll et al., 2010). As for the advantage of $\mathrm{Cot} / \mathrm{CO}$, it can be measured easily and non-invasively. Although, it should be noted that it was not stable over time.

This study had some strong and weak points, which are worth our attention. The main weakness was excluding women due to the marked differences in nicotine metabolism. Also, the study had a small sample size caused by limited human and financial resources. The use of private online counseling did not consume a lot of time and space to contact the subjects under treatment. Ubiquitous access to the therapist and updated data collection via the web were to put a value on patient-therapist interactions and to ensure better analysis of the treatment outcomes. In addition to interactive follow-up, this study applied a biological method to evaluate the patient's progress in quitting attempts and determine their smoking status, which, in turn, positively contributes to personal motivation and treatment adherence.

\section{Conclusions}

This study indicated that $\mathrm{Cot} / \mathrm{CO}$ ratio, as well as $\mathrm{CO} /$ CPD ratio, could predict the smoking cessation success and treatment course among those who received treatments with NP and NL.

\section{Ethical Considerations}

\section{Compliance with ethical guidelines}

All ethical principles are considered in this article. The participants were informed about the purpose of the research and its implementation stages. They were also assured about the confidentiality of their information. They were free to leave the study whenever they wished, and if desired, the research results would be available to them.

\section{Funding}

The Office of Vice -Chancellor for Global Strategies and International Affairs, Tehran University of Medical Sciences supported this study (fund number: 95-01-103-30941). 


\section{Authors' contributions}

All authors equally contributed to preparing this article.

\section{Conflict of interest}

The authors declared no conflict of interest.

\section{References}

2008 PHS Guideline Update Panel, Liaisons, and Staff. (2008) Treating tobacco use and dependence: 2008 update U.S. Public Health Service Clinical Practice Guideline executive summary. Respiratory Care, 53(9), 1217-22. [PMID]

Adams, T. N., \& Morris, J. (2021). Smoking. Treasure Island, FL: StatPearls Publishing. https://www.ncbi.nlm.nih.gov/ books/NBK537066/

Alimohammadi, M., Jafari-Mansoorian, H., Hashemi, S. Y., Momenabadi, V., Ghasemi, S. M., \& Karimyan, K. (2017). Review on the implementation of the Islamic Republic of Iran about tobacco control, based on MPOWER, in the framework convention on tobacco control by the World Health Organization. Addiction \& Health, 9(3), 183-9. [PMID] [PMCID]

Batra, A., Collins, S. E., Torchalla, I., Schröter, M., \& Buchkremer, G. (2008). Multidimensional smoker profiles and their prediction of smoking following a pharmacobehavioral intervention. Journal of Substance Abuse Treatment, 35(1), 41-52. [DOI:10.1016/j.jsat.2007.08.006] [PMID]

Benowitz, N. L. (1999). Biomarkers of environmental tobacco smoke exposure. Environmental Health Perspectives, 107(Suppl 2), 349-55. [DOI:10.1289/ehp.99107s2349] [PMID] [PMCID]

Benowitz, N. L., \& Jacob III, P. (1994). Metabolism of nicotine to cotinine studied by a dual stable isotope method. Clinical Pharmacology \& Therapeutics, 56(5), 483-93. [DOI:10.1038/ clpt.1994.169] [PMID]

Benowitz, N. L., Jacob 3rd, P., Fong, I., \& Gupta, S. (1994). Nicotine metabolic profile in man: Comparison of cigarette smoking and transdermal nicotine. The Journal of Pharmacology and Experimental Therapeutics, 268(1), 296-303. [PMID]

Benowitz, N. L., Lessov-Schlaggar, C. N., Swan, G. E., \& Jacob III, P. (2006). Female sex and oral contraceptive use accelerate nicotine metabolism. Clinical Pharmacology \& Therapeutics, 79(5), 480-8. [DOI:10.1016/j.clpt.2006.01.008] [PMID]

Berlin, I., Singleton, E. G., \& Heishman, S. J. (2013). Predicting smoking relapse with a multidimensional versus a single-item tobacco craving measure. Drug and Alcohol Dependence, 132(3), 513-20. [DOI:10.1016/j.drugalcdep.2013.03.017] [PMID]

Bough, K. J., Lerman, C., Rose, J. E., McClernon, F. J., Kenny, P. J., \& Tyndale, R. F., et al. (2013). Biomarkers for smoking cessation. Clinical Pharmacology \& Therapeutics, 93(6), 526-38. [DOI:10.1038/clpt.2013.57] [PMID] [PMCID]
Brock, A. J., Takeda, A., Brennan, C., \& Walton, R. T. (2011). Treatment for tobacco dependence: A potential application for stratified medicine? Personalized Medicine, 8(5), 571-9. [DOI:10.2217/pme.11.60] [PMID]

Cahill, K., Lindson-Hawley, N., Thomas, K. H., Fanshawe, T. R., \& Lancaster, T. (2016). Nicotine receptor partial agonists for smoking cessation. Cochrane Database of Systematic Reviews, (5), CD006103. [DOI:10.1002/14651858.CD006103.pub7] [PMID] [PMCID]

Chenoweth, M. J., Novalen, M., Hawk Jr, L. W., Schnoll, R. A., George, T. P., \& Cinciripini, P. M., et al. (2014). Known and novel sources of variability in the nicotine metabolite ratio in a large sample of treatment-seeking smokers. Cancer Epidemiology, Biomarkers \& Prevention, 23(9), 1773-82. [DOI:10.1158/1055-9965.EPI-14-0427] [PMID] [PMCID]

coVita. (2020). Smokerlyzer®: Helping people to stop smoking one breath at a time. Retrieved from https://www.covita. net/the-smokerlyzer-range/\#literature

de Leon, J., Diaz, F. J., Becoña, E., Gurpegui, M., Jurado, D., \& Gonzalez-Pinto, A. (2003). Exploring brief measures of nicotine dependence for epidemiological surveys. Addictive Behaviors, 28(8), 1481-6. [DOI:10.1016/S0306-4603(02)00264-2]

Dempsey, D., Tutka, P., Jacob III, P., Allen. F., Schoedel. K., \& Tyndale, R. F., et al. (2004). Nicotine metabolite ratio as an index of cytochrome P450 2A6 metabolic activity. Clinical Pharmacology \& Therapeutics, 76(1), 64-72. [DOI:10.1016/j. clpt.2004.02.011] [PMID]

Fogli, S., Saba, A., Del Re, M., Pistelli, F., Aquilini, F., \& Zucchi, R., et al. (2016). Application of a pharmacokinetic/ pharmacogenetic approach to assess the nicotine metabolic profile of smokers in the real-life setting. Journal of Pharmaceutical and Biomedical Analysis, 131, 208-13. [DOI:10.1016/j. jpba.2016.08.041] [PMID]

Hammond, S. K., \& Leaderer, B. P. (1987). A diffusion monitor to measure exposure to passive smoking. Environmental Science E Technology, 21(5), 494-7. [DOI:10.1021/es00159a012] [PMID]

Hartz, S. M., Short, S. E., Saccone, N. L., Culverhouse, R., Chen L. S., \& Schwantes-An, T. H., et al. (2012). Increased genetic vulnerability to smoking at CHRNA5 in early-onset smokers. Archives of General Psychiatry, 69(8), 854-60. [DOI:10.1001/ archgenpsychiatry.2012.124] [PMID] [PMCID]

Haug, S., Schaub, M. P., \& Schmid, H. (2014). Predictors of adolescent smoking cessation and smoking reduction. $\mathrm{Pa}$ tient Education and Counseling, 95(3), 378-83. [DOI:10.1016/j. pec.2014.03.004] [PMID]

Heatherton, T. F., Kozlowski, L. T., Frecker, R. C., \& Fagerstrom, K. O. (1991). The Fagerström test for nicotine dependence: A revision of the Fagerstrom tolerance questionnaire. British Journal of Addiction, 86(9), 1119-27. [DOI:10.1111/j.1360-0443.1991. tb01879.x] [PMID]

Ho, M. K., Faseru, B., Choi, W. S., Nollen, N. L., Mayo, M. S., \& Thomas, J. L., et al. (2009). Utility and relationships of biomarkers of smoking in African-American light smokers Cancer Epidemiology, Biomarkers \& Prevention, 18(12), 3426-34. [DOI:10.1158/1055-9965.EPI-09-0956] [PMID] [PMCID]

Jaakkola, M. S., \& Jaakkola, J. J. (1997). Assessment of exposure to environmental tobacco smoke. European Respiratory Journal, 10(10), 2384-97. [DOI:10.1183/09031936.97.10102384] [PMID] 
Jacob, P., \& Byrd, G. D. (1999). Use of gas chromatographic and mass spectrometric techniques for the determination of nicotine and its metabolites. In J. W. Gorrod, \& P. Jacob III (Eds.) Analytical determination of nicotine and related compounds and their metabolites (pp. 191-224). Amsterdam: Elsevier Science B.V. [DOI:10.1016/B978-044450095-3/50007-3] [PMID]

Jayakrishnan, R., Uutela, A., Mathew, A., Auvinen, A., Mathew, P. S., \& Sebastian, P. (2013). Smoking cessation intervention in Rural Kerala, India: Findings of a randomised controlled trial. Asian Pacific Journal of Cancer Prevention, 14(11), 6797-802. [DOI:10.7314/APJCP.2013.14.11.6797] [PMID]

Jha, P. (2020). The hazards of smoking and the benefits of cessation: A critical summation of the epidemiological evidence in high-income countries. eLife, 9, e49979. [DOI:10.7554/ eLife.49979] [PMID] [PMCID]

Johnstone, E., Benowitz, N., Cargill, A., Jacob, R., Hinks, L., \& Day, I., et al. (2006). Determinants of the rate of nicotine metabolism and effects on smoking behavior. Clinical Pharmacology \& Therapeutics, 80(4), 319-30. [DOI:10.1016/j. clpt.2006.06.011] [PMID]

Jonk, Y. C., Sherman, S. E., Fu, S. S., Hamlett-Berry, K. W., Geraci, M. C., \& Joseph, A. M. (2005). National trends in the provision of smoking cessation aids within the Veterans Health Administration. The American Journal of Managed Care, 11(2), 77-85. [PMID]

Joseph, A. M., Hecht, S. S., Murphy, S. E., Carmella, S. G., Le, C. T., \& Zhang, Y., et al. (2005). Relationships between cigarette consumption and biomarkers of tobacco toxin exposure. Cancer Epidemiology, Biomarkers \& Prevention, 14(12), 2963-8. [DOI:10.1158/1055-9965.EPI-04-0768] [PMID]

Klesges, R. C., Debon, M., \& Ray, J. W. (1995). Are self-reports of smoking rate biased? Evidence from the Second National Health and Nutrition Examination Survey. Journal of Clinical Epidemiology, 48(10), 1225-33. [DOI:10.1016/08954356(95)00020-5]

Kralikova, E., Kmetova, A., Stepankova, L., Zvolska, K., Davis, R., \& West, R. (2013). Fifty-two-week continuous abstinence rates of smokers being treated with varenicline versus nicotine replacement therapy. Addiction, 108(8), 1497-502. [DOI:10.1111/add.12219] [PMID]

Lancaster, T., \& Stead, L. F. (2017). Individual behavioural counselling for smoking cessation. Cochrane Database of Systematic Reviews, (3), CD001292. [DOI:10.1002/14651858.CD001292. pub3] [PMID] [PMCID]

Lea, R. A., Dickson, S., \& Benowitz, N. L. (2006). Within-subject variation of the salivary $3 \mathrm{HC} / \mathrm{COT}$ ratio in regular daily smokers: Prospects for estimating CYP2A6 enzyme activity in large-scale surveys of nicotine metabolic rate. Journal of Analytical Toxicology, 30(6), 386-9. [DOI:10.1093/jat/30.6.386] [PMID]

Mamoun, M., Bergen, A. W., Shieh, J., Wiggins, A., \& Brody, A. L. (2015). Biomarkers of response to smoking cessation pharmacotherapies: Progress to date. CNS Drugs, 29(5), 359-69. [DOI:10.1007/s40263-015-0243-1] [PMID] [PMCID]

Martínez, C., Castellano, Y., Andrés, A., Fu, M., Antón, L., \& Ballbè, M., et al. (2017). Factors associated with implementation of the 5A's smoking cessation model. Tobacco Induced Diseases, 15, 41. [DOI:10.1186/s12971-017-0146-7] [PMID] [PMCID]
Mooney, M. E., Li, Z. Z., Murphy, S. E., Pentel, P. R., Le, Ch., \& Hatsukami, D. K. (2008). Stability of the nicotine metabolite ratio in ad libitum and reducing smokers. Cancer Epidemiology, Biomarkers \& Prevention, 17(6), 1396-400. [DOI:10.1158/10559965.EPI-08-0242] [PMID] [PMCID]

Paluck, E. C., McCormack, J. P., Ensom, M. H., Levine, M., Soon, J. A., \& Fielding, D. W. (2006). Outcomes of bupropion therapy for smoking cessation during routine clinical use. Annals of Pharmacotherapy, 40(2), 185-90. [DOI:10.1345/aph.1G324] [PMID]

Payne, T. J., Smith, P. O., McCracken, L. M., McSherry, W. C., \& Antony, M. M. (1994). Assessing nicotine dependence: A comparison of the Fagerstrom Tolerance Questionnaire (FTQ) with the Fagerstrom Test for Nicotine Dependence (FTND) in a clinical sample. Addictive Behaviors, 19(3), 307-17. [DOI:10.1016/0306-4603(94)90032-9]

Perkins, K. A., Karelitz, J. L., \& Jao, N. C. (2013). Optimal carbon monoxide criteria to confirm 24-hr smoking abstinence. Nicotine \& Tobacco Research, 15(5), 978-82. [DOI:10.1093/ntr/ nts205] [PMID] [PMCID]

Pierce, J. P., \& Gilpin, E. A. (2002). Impact of over-the-counter sales on effectiveness of pharmaceutical aids for smoking cessation. JAMA, 288(10), 1260-4. [DOI:10.1001/jama.288.10.1260] [PMID]

Raja, M., Garg, A., Yadav, P., Jha, K., \& Handa, S. (2016). Diagnostic methods for detection of cotinine level in tobacco users: A review. Journal of Clinical and Diagnostic Research, 10(3), ZE04-6. [DOI:10.7860/JCDR/2016/17360.7423] [PMID] [PMCID]

Rao, Y., Hoffmann, E., Zia, M., Bodin, L., Zeman, M., \& Sellers, E. M., et al. (2000). Duplications and defects in the CYP2A6 gene: Identification, genotyping, and in vivo effects on smoking. Molecular Pharmacology, 58(4), 747-55. [DOI:10.1124/ mol.58.4.747] [PMID]

Rezaei, S., Pulok, M. H., \& Ebrahimi, M. (2020). Socioeconomic inequality in tobacco expenditure in Iran: A cross-sectional analysis at national and subnational levels. BMC Public Health, 20, 1031. [DOI:10.1186/s12889-020-09144-z] [PMID] [PMCID]

Rubinstein, M. L., Shiffman, S., Rait, M. A., \& Benowitz, N L. (2013). Race, gender, and nicotine metabolism in adolescent smokers. Nicotine E Tobacco Research, 15(7), 1311-5. [DOI:10.1093/ntr/nts272] [PMID] [PMCID]

Saidi, O., Hajjem, S., Zoghlami, N., Aounallah-Skhiri, H., Ben Mansour, N., \& Hsairi, M., et al. (2019). Premature mortality attributable to smoking among Tunisian men in 2009. Tobacco Induced Diseases, 17, 77. [DOI:10.18332/tid/112666] [PMID] [PMCID]

Samet, J. M. (2013). Tobacco smoking: The leading cause of preventable disease worldwide. Thoracic Surgery Clinics, 23(2) 103-12. [DOI:10.1016/j.thorsurg.2013.01.009] [PMID]

Sarbandi, F., Niknami, Sh., Hidarnia, A. R., Hajizadeh, E. Azaripour Masooleh, H., \& Eslampanah Nobari, Sh. (2015). Psychometric properties of the Iranian version of the Fagerström test for nicotine dependence and of heaviness of smoking index. Journal of Research and Health, 5(1), 96-103. http:// jrh.gmu.ac.ir/article-1-507-en.html 
Schnoll, R. A., Martinez, E., Tatum, K. L., Glass, M., Bernath, A., \& Ferris, D., et al. (2010). Nicotine patch vs. nicotine lozenge for smoking cessation: An effectiveness trial coordinated by the Community Clinical Oncology Program. Drug and Alcohol Dependence, 107(2-3), 237-43. [DOI:10.1016/j.drugalcdep.2009.11.001] [PMID] [PMCID]

Sharma, P., Sane, N., Anand, S. D., Marimutthu, P., \& Benegal, V. (2019). Assessment of cotinine in urine and saliva of smokers, passive smokers, and nonsmokers: Method validation using liquid chromatography and mass spectrometry. Indian Journal of Psychiatry, 61(3), 270-6. [DOI:10.4103/psychiatry.IndianJPsychiatry_61_18] [PMID] [PMCID]

Shiffman, S., Dresler, C. M., Hajek, P., Gilburt, S. J., Targett, D. A., \& Strahs, K. R. (2002). Efficacy of a nicotine lozenge for smoking cessation. Archives of Internal Medicine, 162(11), 126776. [DOI:10.1001/archinte.162.11.1267] [PMID]

SRNT Subcommittee on Biochemical Verification. (2002). Biochemical verification of tobaccouseand cessation. Nicotine $\mathcal{E}$ Tobacco Research, 4(2), 149-59. [DOI:10.1080/14622200210123581] [PMID]

Stead, L. F., Koilpillai, P., Fanshawe, T. R., \& Lancaster, T. (2016). Combined pharmacotherapy and behavioural interventions for smoking cessation. Cochrane Database of Systematic Reviews, (3), CD008286. [DOI:10.1002/14651858.CD008286.pub3] [PMID]

Wang, C., Cho, B., Xiao, D., Wajsbrot, D., \& Park, P. W. (2013). Effectiveness and safety of varenicline as an aid to smoking cessation: Results of an inter-Asian observational study in real-world clinical practice. International Journal of Clinical Practice, 67(5), 469-76. [DOI:10.1111/ijcp.12121] [PMID]

Waters, A. J., Shiffman, S., Sayette, M. A., Paty, J. A., Gwaltney, C. J., \& Balabanis, M. H. (2004). Cue-provoked craving and nicotine replacement therapy in smoking cessation. Journal of Consulting and Clinical Psychology, 72(6), 1136-43. [DOI:10.1037/0022-006X.72.6.1136] [PMID]

West, R., DiMarino, M. E., Gitchell, J., \& McNeill, A. (2005). Impact of UK policy initiatives on use of medicines to aid smoking cessation. Tobacco Control, 14(3), 166-71. [DOI:10.1136/ tc.2004.008649] [PMID] [PMCID]

World Health Organization. (2019). WHO global report on trends in prevalence of tobacco use 2000-2025. Retrieved from https://www.who.int/publications/i/item/ who-global-report-on-trends-in-prevalence-of-tobaccouse-2000-2025-third-edition 
This Page Intentionally Left Blank 